\title{
iUBICOM '11: The 6th International Workshop on Ubiquitous and Collaborative Computing
}

In conjunction with the 25th BCS Conference on Human Computer Interaction ( $\mathrm{HCl} 2011)$

Northumbria University, Newcastle

4 July 2011 


\section{Abstract}

Following five successful previous iUBICOM workshops, we present the 6th iUBICOM workshop, iUBICOM 11. This year's theme was 'information retrieval, decision making processes and user needs in the context of ubiquitous and collaborative computing.'

A particular focus of this year's workshop was on how user needs can be interpreted given new interaction models and unfamiliar technologies. We discussed the challenge of understanding user needs around factors such as speed, transparency, efficiency and quality of service. Applications included wayfinding systems, smart homes, assistive technologies, personalised information retrieval and collaborative systems. The emphasis was in the consideration of human factors when designing and developing systems that can be deployed successfully. 


\section{Full Synopsis}

Mark Weiser put forth a vision of ubiquitous computing, according to which people and environments are augmented with computational resources which provide information and services whenever and wherever required. Such systems or services feature multi-device architectures and complex interactions. They are often part of the environment, where they host a range of everyday activities in ways that imply new kinds of user involvement. With these sorts of systems, it is important to understand the way systems and people work in the environments which are to be augmented. The purpose of this workshop was to bring multi-disciplinary researchers together in order to discuss different models and theories to design and evaluate ubiquitous and collaborative computing systems and services. Given these issues, we aimed to balance technological innovation with adequate consideration for the needs of users.

Achieving this vision required communication and interoperability between different applications and devices. The availability of information through a wide range of media including mobile devices and sensors has given a new momentum to ubiquitous and collaborative computing by providing people with new opportunities to communicate with geographically dispersed teams and to conduct business.

A particular focus of this year's workshop was on how user needs can be interpreted given new interaction models and unfamiliar technologies. We discussed the challenge of understanding user needs around factors such as speed, transparency, efficiency and quality of service. Applications included wayfinding systems, smart homes, assistive technologies, personalised information retrieval and collaborative systems. The emphasis was in the consideration of human factors when designing and developing systems that can be deployed successfully.

The workshop attracted a range of high-quality submissions from researchers working in the areas ranging from semantic technologies through collaborative and supportive systems to knowledge management. This proceeding consists of 13 high-quality papers.

The first paper, by Yuqing Mao, Haifeng Shen and Chengzheng Sun, presents an approach to integrate social networking into a novel epistemology-based social search framework. A prototype system is developed to demonstrate the usefulness of the proposed approach. The experimental results show that the proposed social-network-optimised social search system outperforms traditional search engines.

The second paper, by Fazilatur Rahman and Jawed Siddiqi, presents a semi-automatic semantic annotation approach for digital music. The approach enables music producers to generate music metadata through a mapping between music consumers' free form tags and the acoustic metadata that are automatically extracted from music audio.

The third paper, by Lian Hoy Lee, Christof Lutteroth and Gerald Weber, discusses the need for greater search capabilities that produce relevant search results based on semantic technologies. The authors propose a new platform in order to cater for semantic user interface development as separate modules for Wikipedia. The effectiveness of the proposed approach was tested by implementing several semantic user interface modules.

The fourth paper, by Faiyaz Doctor, Rahat Iqbal and Raouf N. G. Naguib, proposes an ambient intelligent framework for residential monitoring of dementia patients using fuzzy agents. Within this framework groups of unobtrusive wireless sensor devices can be deployed at specific locations within a patient's 
home and accessed via standardized interfaces provided through an open middleware platform. The proposed system reports behaviour changes to care providers to enable them to make better-informed assessments of the patient's cognitive abilities and changing care needs.

The fifth paper, by Ammar Zayouna, Richard Comley and Daming Shi, discusses the need for local-global optical flow for image registration purpose in order to match two or more images taken at different times from different sensors or from different viewpoints. The proposed approach integrates both the local optical flow and global optical flow for image registration. Experiments results are included in the paper to demonstrate the effectiveness of the proposed approach.

The sixth paper, Mark Reilly and Haifeng Shen, discusses the design and implementation of the smart phone based GroupNotes app for ubiquitous and collaborative learning. The GroupNotes allows a small group of students to participate in a real-time collaborative note-taking session using their own smart phones in the lecture.

The seventh paper, Mark Reilly and Haifeng Shen, continues the previous work to extend the GroupNotes app in order to develop a student centred collaborative learning pedagogy into the lecture environment through a novel real-time collaborative note-taking application. The GroupNotes helps to encourage students to proactively engage themselves through smartphones interaction.

The eighth paper, by Adam Grzywaczewski, Rahat Iqbal, Anne James and John Halloran, discusses the requirements for the development of intelligent recommender systems for software developers. The paper discusses a systematic approach that can be used to develop dedicated and personalised search utilities. The paper discusses the results of two user studies: feasibility study and automated capture of software developers' behaviour. Both user studies show that copy and paste can be used as implicit feedback indicator for the development of intelligent recommender systems for software developers in order to support their task.

The ninth paper, by Catherine Mulwa, Luca Longo, Seamus Lawless, Mary Sharp and Vincent Wade, presents an online framework for supporting the evaluation of personalised information retrieval systems. The paper proposes a web-based interface which provides developers and evaluators with implicit recommendations on how to evaluate a specific personalised information retrieval system.

The tenth paper, by Babak Akhgar, Esmaeil Salahi Parvin and Mohammad Hussein Sherkat, presents agile strategic information systems based on axiomatic agent architecture. Strategic information systems in which strategic decisions are made continuously can perform better by employing an axiomatic design approach. The proposed approach emphasises the use of agents for strategic information systems.

The eleventh paper, by Artemis Akhgar and Aryan Gholipour, discusses the role of socialisation in knowledge management in virtual teams. The paper discusses key factors concerning the role of socialisation in improving knowledge creation and knowledge sharing in virtual teams. The quantitative results show a significant correlation between socialisation, tactics and virtual teams.

The twelfth paper, by Ali Atashi and Mahsa Parvini, applies knowledge extracted from communication with customers in a mass customisation process. The paper discusses the role of human-computer interaction in mass customisation processes.

The thirteenth paper, by Mohammad Hassanzadeh, Babak Akhgar and Simeon Yates, presents a knowledge management infrastructure for governmental ubiquitous services. The paper discusses the results of empirical research focusing on the investigation of a knowledge management infrastructure in 
government departments and levels of user-centred architecture for effective deployment of knowledge management.

\section{Workshop Chairs}

Dr. Rahat Iqbal, Coventry University, UK

Professor Jacques Terken, Technical University of Eindhoven, Netherlands

Dr. Dzmitry Aliakseyeu, Philips Research Europe, Netherlands

Professor Anne James, Coventry University, UK 


\section{Papers:}

Yuqing Mao, Haifeng Shen and Chengzheng Sun Google+Facebook: A Social-Network-Optimized Web Search Approach http://dx.doi.org/10.14236/ewic/IUBICOM2011.1

Fazilatur Rahman and Jawed Siddiqi Semi-automatic Semantic Annotation Tool for Digital Music http://dx.doi.org/10.14236/ewic/IUBICOM2011.2

Lian Hoy Lee, Christof Lutteroth and Geral Weber Enhancing Wikipedia with Semantic

Technologies http://dx.doi.org/10.14236/ewic/IUBICOM2011.3

Faiyaz Doctor, Rahat Iqbal and Raouf N.G. Naguib An Ambient Intelligent Framework for

Residential Monitoring of Dementia Patients using Fuzzy Agents

http://dx.doi.org/10.14236/ewic/IUBICOM2011.4

Ammar Zayouna, Richard Comley and Daming Shi Local-Global Optical Flow for Image Registration http://dx.doi.org/10.14236/ewic/IUBICOM2011.5

Mark Reilly and Haifeng Shen The Design and Implementation of the Smartphone-based GroupNotes App for Ubiquitous and Collaborative Learning http://dx.doi.org/10.14236/ewic/IUBICOM2011.6

Mark Reilly and Haifeng Shen Unobtrusive Student Collaboration during Lectures with Smartphones http://dx.doi.org/10.14236/ewic/IUBICOM2011.7

Adam Grzywaczewski, Rahat Iqbal, Anne James and John Halloran Software Developers'

Information Needs: Towards the Development of Intelligent Recommender Systems http://dx.doi.org/10.14236/ewic/IUBICOM2011.8

Catherine Mulwa, Luca Longo, Séamus Lawless, Mary Sharp and Vincent Wade An Online Framework for Supporting the Evaluation of Personalised Information Retrieval Systems

http://dx.doi.org/10.14236/ewic/IUBICOM2011.9

Babak Akhgar, Easmaeil Salahi Parvin and Mohammad Hussein Sherkat Agile Strategic Information Systems based on Axiomatic Agent Architecture http://dx.doi.org/10.14236/ewic/IUBICOM2011.10

Artemis Akhgar and Aryan Gholipour The role of socialization in Knowledge management in virtual teams http://dx.doi.org/10.14236/ewic/IUBICOM2011.11

Ali Atashi and Mahsa Parvini Applying knowledge extracted from communicating with customers in Mass Customization process: the role of Human-Computer Interaction http://dx.doi.org/10.14236/ewic/IUBICOM2011.12

Mohammad Hassanzadeh, Babak Akhgar and Simeon Yates Knowledge Management infrastructure for Governmental Ubiquitous Services http://dx.doi.org/10.14236/ewic/IUBICOM2011.13 